UBCTP-92-24

UCSBTH-92-24

\title{
Driving Operators Relevant: A Feature of Chern-Simons Interaction
}

\author{
Wei Chen* \\ Department of Physics, University of British Columbia \\ Vancouver, B.C. Canada V6T 1Z1 \\ Miao $\mathrm{Li}^{\dagger}$ \\ Department of Physics, University of California \\ Santa Barbara, CA 93106 USA
}

September 1992

\begin{abstract}
By computing anomalous dimensions of gauge invariant composite operators $(\bar{\psi} \psi)^{n}$ and $\left(\phi^{*} \phi\right)^{n}$ in Chern-Simons fermion and boson models, we address that Chern-Simons interactions make these operators more relevant or less irrelevant in the low energy region. We obtain a critical Chern-Simons fermion coupling, $\frac{1}{\kappa_{c}^{2}}=\frac{6}{19}$, for a phase transition at which the leading irrelevant four-fermion operator $(\bar{\psi} \psi)^{2}$ becomes marginal, and a critical Chern-Simons boson coupling, $\frac{1}{\kappa_{c}^{2}}=\frac{6}{34}$, for a similar phase transition for the leading irrelevant operator $\left(\phi^{*} \phi\right)^{4}$. We see this phenomenon also in the $1 / N$ expansion.
\end{abstract}

\footnotetext{
0* chen@physics.ubc.ca

${ }^{0 \dagger}$ Present address: Department of Physics, Brown University, Providence, RI 02912 USA. li@het.brown.edu
} 
One of the remarkable features of Chern-Simons matter gauge theories [1] is that the Chern-Simons interaction attaches statistical flux tubes to particles, by which a fermion can be transmuted into a boson or an anyon (and vise versa), depending on the strength of the Chern-Simons coupling, $1 / \kappa$ ( $\kappa$ the statistical parameter) [2]. In the relativistic quantum field theory scheme, an Abelian Chern-Simons term receives no correction from interacting with massive matters beyond one loop [3] [4], and receives only finite corrections from massless matters starting from two loops [4] [5]. This results in an identically vanishing beta function for Chern-Simons couplings, as the theories are gauge invariant. The insensibility of Chern-Simons couplings to energy scales is attributed to the topological nature of Chern-Simons actions. However, it doesn't imply a triviality of the whole theory in the sense of renormalization. In fact, as shown in [6], matter fields in Chern-Simons models do need infinite renormalization and receive anomalous dimensions 円. This observation leads naturally to a conjecture that asymptotic behavior of gauge invariant operators in a Chern-Simons matter theory is non-trivial. Recently, Chern-Simons boson and fermion models have been used in describing the phase transition between quantum Hall states and insulators in [7] and [8], respectively, where among other observations is that the composite mass operators of matter fields receive as well an anomalous dimension, and therefore not only the critical exponent $\eta$ but also $\nu$ is modified by the Chern-Simons interaction.

In the present letter, we shall address another feature of Chern-Simons matter interaction, namely it modifies the scaling dimensions of a class of gauge invariant composite operators $(\bar{\psi} \psi)^{n}$ of fermion model and $\left(\phi^{*} \phi\right)^{n}$ of boson model, with $n=$ positive integers, in such a way that these operators are driven more relevant or less irrelevant (in the lower energy region).

By dimensional analysis, not many operators in a Chern-Simons Matter model are relevant. For instance, the Euclidean action of a Chern-Simons fermion model, if contains the relevant and marginal operators only, reads

$$
S=\int d^{3} x\left(\bar{\psi} \gamma \cdot \partial \psi+m \bar{\psi} \psi+\bar{\psi} \gamma \cdot A \psi+i \frac{\kappa}{4 \pi} \epsilon^{i j k} A_{i} \partial_{j} A_{k}\right) .
$$

\footnotetext{
${ }^{1}$ Non-Abelian Chern-Simons field receives an anomalous dimension through interacting with matters as well [6], while the Chern-Simons coefficient keeps finite and quantized, as required by the "large" gauge symmetry.
} 
The action (11) is so normalized that $\kappa$ is dimensionless. In particular, the four-fermion operator $(\bar{\psi} \psi)^{2}$ is irrelevant as it has an engineering dimension four; and the Maxwell term $F^{2}$ ( $F$ the field strength), the kinetic of a dynamic gauge field, is irrelevant, compared to the kinetic Chern-Simons $A d A$. Now, an interesting question is how will these be changed by quantum corrections? The answer to the operator $F^{2}$ is obvious: it remains irrelevant, as the dimension of the primary gauge field $A$ doesn't change during the quantization. However, the radiation correction from matters induces a non-local operator concerning the gauge field $A$ in the effective Lagrangian. In the case of massless matters for instance, this induced term is $F\left(1 / \sqrt{\partial^{2}}\right) F$. It is finite and marginal. On the other hand, the operator $(\bar{\psi} \psi)^{2}$ and other gauge invariant composite operators $(\bar{\psi} \psi)^{n}$ are expected to have an anomalous dimension. One of the main results of the present work is the scaling dimensions of such a class of composite operators at the lowest non-trivial order

$$
d_{(\bar{\psi} \psi)^{n}}=2 n-\frac{2 n+15}{6 \kappa^{2}}+O\left(\frac{1}{\kappa^{4}}\right) .
$$

(2) shows that the Chern-Simons interaction makes the mass operator $(n=1)$, already relevant without the Chern-Simons interaction, even more relevant. Its consequences in some phase transitions have been discussed in [8]. Moreover, also due to the ChernSimons interaction, the irrelevant operators $(\bar{\psi} \psi)^{n}(n \geq 2)$ are less irrelevant. The most important operator of this type is the leading irrelevant one, with $n=2$. (2) suggests a phase transition with a critical effective coupling

$$
1 / \kappa_{c}^{2}=6 / 19
$$

at which the leading irrelevant four-fermion operator $(\bar{\psi} \psi)^{2}$ becomes marginal. The critical value in (3) shows that if such a phase transition would occur, it occurs only in a region with rather strong Chern-Simons interaction. We make several remarks at this point. First, it is not difficult to check that, at the order $1 / k^{3}$, all associated Feynman diagrams are finite, the next leading contributions to the anomalous dimensions are from the order $1 / \kappa^{4}$ (four and higher loops), as indicated in (2)). According to (3), therefore, there exists a multiplicative factor $1 / \kappa_{c}^{4} \simeq 1 / 10$ in the next order. If the numerical coefficient of the next order would be smaller than or comparable 
to that of the leading order, the perturbation expansion near the transition point is, to some extent, acceptable. Our second remark is that, once such a phase transition happens, a four-fermion operator, now marginal or relevant, is switched on in the system. In turn, this four-fermion self-interaction develops a positive anomalous dimension [9] that has the potential to make the operator irrelevant. As a result of competition between the Chern-Simon-fermion and four-fermion interactions, a balance would be reached somewhere. The last, a bare four-fermion interaction in three dimensions has a coupling constant that carries a negative dimension of mass and this makes the operator non-renormalizable. However, once the Chern-Simons interaction turns the operator marginal or relevant, the effective coupling of the four-fermion interaction has zero or a positive dimension and therefore the four-fermion operator becomes renormalizable or super-renormalizable.

It is equally interesting to conduct a parallel discussion for the Chern-Simons boson model. In this model, as the scalar field in three dimensions has an engineering dimension $1 / 2$, the boson self-interactions $g_{1}\left(\phi^{*} \phi\right)^{2}$ and $g_{2}\left(\phi^{*} \phi\right)^{3}$ are relevant and marginal, respectively, and therefore should be taken into account. However, if one perturbs the theory near the Gaussian (ultra-violet) fixed point of these interactions, where $g_{1} \sim g_{2} \sim 0$, the boson self-interactions can be turned off for the time being. Assuming so, similar to (2), we obtain the scaling dimensions for the operators $\left(\phi^{*} \phi\right)^{n}$

$$
d_{\left(\phi^{*} \phi\right)^{n}}=n-\frac{7 n+6}{6 \kappa^{2}}+O\left(\frac{1}{\kappa^{4}}\right) .
$$

Same to what happens in the fermion model, the Chern-Simons interaction decreases the scaling dimensions of these operators. The critical Chern-Simons coupling for a phase transition at which the leading irrelevant operator $\left(\phi^{*} \phi\right)^{4}$ becomes marginal is

$$
1 / \kappa_{c}^{2}=3 / 17
$$

On the other hand, if a perturbation is performed near the infrared fixed points of the self-interactions $\left(\phi^{*} \phi\right)^{2}$ and/or $\left(\phi^{*} \phi\right)^{3}$, one can not switch off them, as the critical couplings $g_{1}^{*}$ and $g_{2}^{*}$ can be rather strong and they are the driving forces near the infrared fixed points. The self-interactions contribute to (4) positive terms that increase the scaling dimensions of the operators. The self-interaction of $\left(\phi^{*} \phi\right)^{2}$, 
for example, at the infrared fixed point may be so strong that it alone makes itself irrelevant. However, a sufficiently strong Chern-Simons interaction may possibly draw it back, and the corresponding infrared fixed point is significantly shifted.

Another interesting case is the Chern-Simons matter system involving $N$ spices of matters with some symmetry, $O(N)$ for instance. A possible perturbation expansion in this case is over $1 / N$. We shall consider this expansion and see the same phenomenon at the order $O(1 / N)$, just before ending the letter.

Now we turn to renormalization. Though, in a procedure of renormalization, one normally deals with the ultra-violet divergences, the resulting (ultra-violet) finite effective theory takes a form that is equally good in exhibiting the asymptotic behavior of the theory in both high and low energy limits. Let us take a simple example. After renormalization, the two-point function of fermion in the momentum space has an asymptotic form $S(p)=<\psi(p) \bar{\psi}(-p)>=\frac{1}{i \gamma \cdot p}\left(\frac{p^{2}}{\mu^{2}}\right)^{\gamma_{\psi}}$, where $\mu$ is a reference mass parameter and $\gamma_{\psi}$ the anomalous dimension of the fermion field. In the Chern-Simons fermion model, $\gamma_{\psi}=-\frac{1}{3 \kappa^{2}} \leq 0$, at the lowest non-trivial order and in the Landau gauge[6]. This implies that the fermion field in th Chern-Simons quantum field theory has a dimension less than its engineering one. Moreover, the kinetic term $\bar{\psi} \gamma \cdot \partial \psi$ takes an asymptotic form $\left(\frac{p^{2}}{\mu^{2}}\right)^{\gamma_{\psi}}$. Now we see the kinetic of fermion in the Chern-Simons theory is relevant in the lower energy region, instead of marginal.

Though there are several independent terms in the action (1), to renormalize it, only one non-trivial renormalization constant suffices. This is because $Z_{A}=1$ can always be chosen, as the Abelian gauge field $A$ needs no infinite renormalization, and $Z_{\psi}=Z_{(\bar{\psi} A \psi)}$, due to the gauge symmetry. The fermion wave-function renormalization constant, to the lowest non-trivial order, in the Landau gauge, and under the minimal subtraction, is [6]

$$
Z_{\psi}=1+\frac{1}{3 \kappa^{2}} \frac{1}{\epsilon}
$$

with $\epsilon=3-\omega \rightarrow 0$. Now we consider the composite operators of interest. For simplicity, we shall set matter mass to zero $(m=0)$. This will not change the ultra-violet divergence structure of the model. We shall use the Landau gauge for convenience, the results must be independent of a gauge choice as we are dealing with 
the gauge invariant operators. The regularization by dimensional reduction, as used in [6] [8], will be used here. The same results have been obtained by introducing a naive cutoff, as a check of consistency. To calculate the renormalization of a composite operator $O_{n}=\frac{1}{(n !)^{2}}(\bar{\psi} \psi)^{n}$ for a given $n$, we construct an 1PI composite vertex $\Gamma_{O_{n}}^{2 n}$ which contains the operator $O_{n}$ as a vertex and has $n$ truncated external fermion and $n$ anti-fermion (and null Chern-Simons) lines. The non-trivial Feynman diagrams at the lowest non-trivial order are listed in Fig. 1.
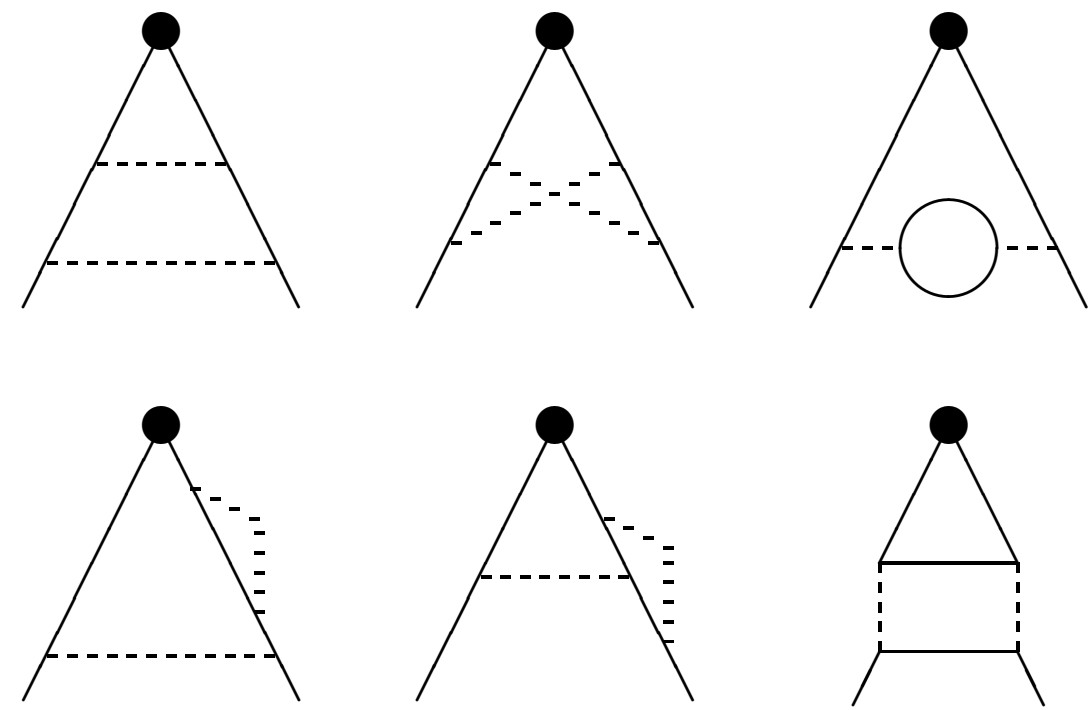

Fig. 1 Non-trivial Feynman diagrams for $\Gamma_{O_{n}}^{2 n}$ at the order $O\left(1 / \kappa^{2}\right)$ in the fermion model. Real line stands for the fermion propagator; dashed line the ChernSimons propagator; and dark spot the operator $O_{n}=\frac{1}{(n !)^{2}}(\bar{\psi} \psi)^{n}$ with $2(n-1)$ external fermion lines omitted. A symmetric factor two for each of the last three diagrams.

By power counting, the composite vertex $\Gamma_{O_{n}}^{2 n}$ is dimensionless. The calculation is somehow tedious but straightforward. The result turns out to be

$$
\Gamma_{O_{n}}^{2 n}\left(\mathbf{0}, p ; \frac{1}{\epsilon}\right)=1+\left[\frac{5}{2 \kappa^{2}}\left(\frac{1}{\epsilon}\right)+\ln \left(\frac{\mu^{2}}{p^{2}}\right)+\text { finite }\right],
$$

where, without loss of generality, we have set all external momenta but one zero. 
Then using the renormalization relation

$$
\left(\Gamma_{O_{i}}^{2 n}\right)_{R}(p)=Z_{\psi}^{n}\left(\frac{1}{\epsilon}\right)\left(Z_{O}\right)_{i j}\left(\frac{1}{\epsilon}\right) \Gamma_{O_{j}}^{2 n}\left(p ; \frac{1}{\epsilon}\right)
$$

and the fermion wave-function renormalization constant (6), we obtain the renormalization constant and anomalous dimension for the operator $(\bar{\psi} \psi)^{n}$

$$
\begin{aligned}
Z_{(\bar{\psi} \psi)^{n}} & =1-\frac{2 n+15}{6 \kappa^{2}}\left(\frac{1}{\epsilon}\right), \\
\gamma_{(\bar{\psi} \psi)^{n}} & =-\frac{2 n+15}{6 \kappa^{2}} .
\end{aligned}
$$

It is worth to notice a simplicity appeared here. As shown in the renormalization relation (8), in its general form, there may exist operator mixing in renormalization of higher dimensional operators. The operator mixing, if happens, will make the situation much more complicated. However, operator mixing does not appear in the renormalization of the class of operators $(\bar{\psi} \psi)^{n}$ of the Chern-Simons fermion model, as we have seen; neither in that of $\left(\phi^{*} \phi\right)^{n}$ of the Chern-Simons boson model, as we shall see, at least in the lowest non-trivial order. This is because the only primary divergence brought by the operator $(\bar{\psi} \psi)^{n}$ for a given $n$ is that in (7). In other words, any cutoff dependence associated with the vertex $O_{n}$ can be cured by just one counterterm associated with (9). We have calculated another composite vertex with $O_{n}, \Gamma_{O_{n}}^{2(n-1)}$, and confirmed that there is indeed no need for any new counterterm to cure the divergence - the one given in (9) suffices - though the composite vertex $\Gamma_{O_{n}}^{2(n-1)}$ has dimension two and may have various divergences.

In the Chern-Simons boson model, the gauge invariant composite operators under consideration are $O_{n}=\frac{1}{(n !)^{2}}\left(\phi^{*} \phi\right)^{n}$, which have an engineering dimension $n$. To perform the renormalization and therefore to calculate their anomalous dimensions, we consider similarly the composite vertex $\Gamma_{O_{n}}^{2 n}$ with the operator $O_{n}$ and $n$ external charged boson and $n$ anti-charged boson lines. The life here seems easier as, at the lowest non-trivial order, there is only one non-trivial diagram, as shown in Fig. 2, for $\Gamma_{O_{n}}^{2 n}$. 


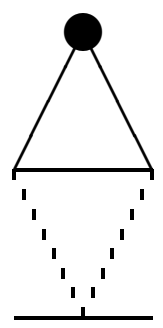

Fig. 2 The only non-trivial Feynman diagram for $\Gamma_{O_{n}}^{2 n}$ at the order $O\left(1 / \kappa^{2}\right)$ in the boson model. Real line stands for the boson propagator; dashed line the Chern-Simons propagator; and dark spot the operator $O_{n}=\frac{1}{(n !)^{2}}\left(\phi^{*} \phi\right)^{n}$ with $2(n-1)$ external boson lines omitted. There is a symmetric factor two for the diagram.

Calculating Fig. 2, we obtain

$$
\Gamma_{O_{n}}^{2 n}\left(\mathbf{0}, p ; \frac{1}{\epsilon}\right)=1+\left[\frac{1}{\kappa^{2}}\left(\frac{1}{\epsilon}\right)+\ln \left(\frac{\mu^{2}}{p^{2}}\right)+\text { finite }\right] .
$$

Finally, we have the renormalization constant and anomalous dimension of $\left(\phi^{*} \phi\right)^{n}$

$$
\begin{aligned}
Z_{\left(\phi^{*} \phi\right)^{n}} & =1-\frac{7 n+6}{6 \kappa^{2}}\left(\frac{1}{\epsilon}\right), \\
\gamma_{\left(\phi^{*} \phi\right)^{n}} & =-\frac{7 n+6}{6 \kappa^{2}} .
\end{aligned}
$$

To get these, we have used the boson model version of the renormalization relation (8) and the boson wave-function renormalization constant $Z_{\phi}=1+\frac{7}{6 \kappa^{2}}\left(\frac{1}{\epsilon}\right)$ [6].

To conclude this letter, we discuss the $1 / N$ expansion, assuming there are $N$ species of matter fields which obey a global symmetry $O(N)$. We shall see the same phenomenon that the Chern-Simons interaction makes the composite operators of interest more relevant or less irrelevant. We take the fermion model as an example and it is straightforward to generalize to the boson model. As the one-loop fermion bubble chain is at the same $O\left(1 / N^{0}\right)$ order with the bare Chern-Simons propagator, one must use a dressed gauge propagator that sums over the one-fermion-loop chains, 
instead of the bare one, in the $1 / N$ expansion. The dressed gauge propagator in the Landau gauge takes the form, same in both the fermion and boson models,

$$
\begin{aligned}
\Delta^{\mu \nu}(p) & =A \frac{\delta^{\mu \nu} p^{2}-p^{\mu} p^{\nu}}{p^{3}}+B \frac{\epsilon^{\mu \nu \lambda} p^{\lambda}}{p^{2}}, \\
A & =\frac{8 \pi \theta}{64 \theta^{2}+\pi^{2}}, \quad B=-\frac{64 \theta^{2}}{64 \theta^{2}+\pi^{2}},
\end{aligned}
$$

where $1 / \theta$ is the effective Chern-Simons coupling. The fermion wave-function renormalization constant at the order $O(1 / N)$ and in the Landau gauge is 8

$$
Z_{\psi_{i}}=1+\frac{16}{3 \pi \theta\left(64 \theta^{2}+\pi^{2}\right) N}\left(\frac{1}{\epsilon}\right)
$$

The non-trivial diagrams, at the same $O(1 / N)$ order, for the composite vertex $\Gamma_{O_{n}}^{2 n}$ with the gauge invariant composite operator $\frac{1}{(n !)^{2}}\left(\bar{\psi}_{i} \psi_{i}\right)^{n}$ are given in Fig. 3.

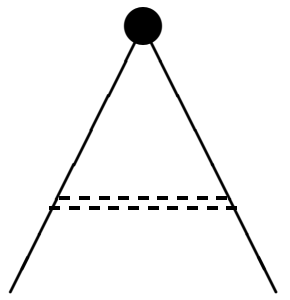

(a)

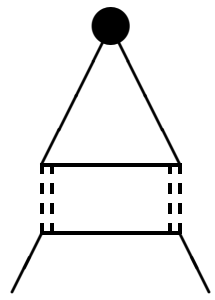

(b)

Fig. 3 Non-trivial Feynman diagram of $\Gamma_{O_{n}}^{2 n}$ at the order $O(1 / N)$. Real line stands for the fermion propagator; double dashed line the dressed gauge propagator; and dark spot the operator $O_{n}=\frac{1}{(n !)^{2}}\left(\bar{\psi}_{i} \psi_{i}\right)^{n}$ with $2(n-1)$ external lines omitted. There is a symmetric factor two for the diagram $(b)$.

Calculating the diagrams in Fig. 3, and using (8), (15) and (16), we obtain at the order $O(1 / N)$

$$
\gamma_{(\bar{\psi} \psi)^{n}}=-\frac{16\left(64 n \theta^{2}+n \pi^{2}+96 \theta^{2}-9 \pi^{2}\right)}{3\left(64 \theta^{2}+\pi^{2}\right)^{2}} \frac{1}{N} .
$$

Now we see the anomalous dimensions of $(\bar{\psi} \psi)^{n}$ are negative and therefore these operators are more relevant (for $n=1$ ) or less irrelevant (for $n \geq 2$ ), only if the 
Chern-Simons coupling is not unreasonably strong, not stronger than $1 / \theta^{2} \sim 15$ for $n=2$ for instance.

The authors would thank I. Affleck, J.-W. Gan, G. Semenoff, Y.-S. Wu for discussions. Work of W.C. was supported in part by the Natural Sciences and Engineering Research Council of Canada. Work of M.L. was supported by DOE grant DOE-76ER70023. Part of this work was done during M.L. visiting Aspen Center for Physics, its hospitality is gratefully acknowledged.

\section{References}

[1] S. Deser, R. Jackiw and S. Templeton, Phys. Rev. Lett. 48 (1982) 975; Ann. Phys. (N.Y.) 140 (1982) 372.

[2] See, for instance, A. Polyakov, Modn. Phys. Lett. A3 (1988) 325.

[3] S. Coleman and B. Hill, Phys. Lett. 159B (1985) 184.

[4] G.W. Semenoff, P. Sodano and Y.-S. Wu, Phys. Rev. Lett. 62 (1989) 715.

[5] W. Chen, Phys. Lett. 251B (1990) 415; V.P. Spridonov, JETP Lett. 52 (1990) No.10, 112; V.P. Spiridonov and F.V. Tkachov, Phys. Lett. B260 (1991) 109.

[6] W. Chen, G.W. Semenoff and Y.-S. Wu, Phys. Rev. D44 (1991) R1625; TwoLoop Analysis of Non-abelian Chern-Simons Theory (Phys. Rev. D, in press).

[7] X.-G. Wen and Y.-S. Wu, Transitions Between the Quantum Hall States and Insulators Induced by Periodic Potentials, preprint (1992).

[8] M.P.A. Fisher, W. Chen and Y.-S. Wu, Mott Transition in An Anyon Gas, UBCTP 92-28, MIT-CTP\# 2157.

[9] W. Chen, Y. Makeenko and G.W. Semenoff, Conformal Bootstrap for Fourfermion Interaction, UBCTP 92-30. 